\title{
Construction of Galois Fields of Characteristic Two and Irreducible Polynomials
}

\author{
By J. D. Swift
}

1. Introduction. The primary purpose of this paper is to provide a practical method of constructing Galois fields of characteristic 2 of large orders and thereby simultaneously give a practical way of generating a large supply of polynomials of high degree irreducible modulo 2 . These polynomials are of special interest in connection with the theory of linear recursive sequences. See, for example [5]; some applications are suggested in [t].

The basic structure of Galois fields is extremely simple. For each prime $q$ and each $n$ there is one and (up to isomorphism) only one finite field of order $q^{n}$, designated by $G F\left(q^{n}\right)$. Its additive group is the elementary abelian group; the direct sum of $n$ cyclic groups of order $q$. The multiplicative group of the non-zero elements is cyclic. The field $G F\left(q^{n m}\right)$ may be constructed from a given $G F\left(q^{n}\right)$ by finding a polynomial of degree $m$, irreducible over $G F\left(q^{n}\right)$ and considering the set of polynomials with coefficients in $G F\left(q^{n}\right)$ modulis $q$ and the irreducible polynomial. The subfields of $G F\left(q^{n}\right)$ are precisely $G F\left(q^{d}\right)$ where $d \mid n$; if $g$ is a primitive generator of $G F\left(q^{n}\right)$, i.e. of the multiplicative subgroup of its non-zero elements, $g^{m}$ is a primitive generator of $G F\left(q^{d}\right)$ where $m=\left(q^{n}-1\right) /\left(q^{d}-1\right)$.

These theorems, covered by many basic texts of algebra since the original work of L. E. Dickson [2], essentially dispose of the elementary theory of these fields. A particularly complete theory is contained in [1] which also has a bibliography listing a number of items from the extensive literature of the deeper arithmetic of the Galois fields. In general, results quoted without proof in this paper may either be found directly in [1] or are immediate consequences of statements proved therein.

From a practical standpoint, the only problems left by the structure theorems are those of finding an irreducible polynomial of degree $m$ over the base field and of finding a primitive generator of the field with respect to this polynomial. In certain cases an irreducible polynomial is readily available and we are here concerned with the exploitation of these cases.

2. Cyclotomic polynomials over $G F(2)$. Let $p$ be a prime for which 2 is a primitive root; then the cyclotomic polynomial $f_{p}(x)=\left(x^{p}+1\right) /(x+1)$ is irreducible over $G F(2)$. Thus for such primes the theory permits the realization of $G F\left(2^{p-1}\right)$. Further, if $g$ is a primitive generator of this field we may realize $G F\left(2^{d}\right)$ by considering powers of $g^{m}, m=\left(2^{p-1}-1\right) /\left(2^{d}-1\right)$ when $d !(p-1)$. Since the only obvious restriction imposed on $p$ by the condition that 2 be a primitive root of $p$ is that $p \equiv \pm 3(\bmod 8)$, it is likely that all fields $G F\left(2^{n}\right), n \not \equiv 0(\bmod 8), \operatorname{may}$ be realized in this way for sufficiently large $p$. The smallest fields which cannot be constructed from polynomials listed in this paper are those for which $n=8,16,17$.

If $z$ is any element of $G F\left(2^{n}\right)$ the powers $z^{0}, z^{1}, \cdots, z^{n}$ will be linearly dependent over $G F(2)$ and the resulting relation of dependence $f(z)=0$ will give an irreducible

Received October 19, 1959. 
polynomial $f(z)$ if $z$ does not lie in a proper subfield of $G F\left(2^{n}\right)$. If $z$ is a primitive generator of $G F\left(2^{n}\right), f(z)$ will be, by definition, primitive irreducible. All irreducible polynomials over $G F(2)$ may be constructed in this way.

By simple counting arguments we see that the number of irreducible polynomials of degree $n$ is

$$
\frac{1}{n}\left(2^{n}-\Sigma 2^{n / q_{i}}+\Sigma 2^{n / q_{i} q_{i}}-\cdots\right)
$$

where the $q_{i}$ are the distinct prime divisors of $n$. Similarly, the number of primitive irreducible polynomials of this degree is $(1 / n) \varphi\left(2^{n}-1\right)$ where $\varphi$ is the Euler totient.

The actual problem of construction of these fields now reduces to finding a generator of the cyclic group. It is at this point that the theory carries us no further and resort must be taken to high speed computing machinery. It is easy to see that, for $p>5$, any generator modulo the cyclotomic polynomial must be of degree at least 3. In particular, since $x^{p}=1, x$ and $x^{2}$ are of order $p$. To investigate the orders of the other linear and quadratic forms we study $z=x+1 / x$; define $h(z)=$ $x^{-m} f_{p}(x)$ where $m=(p-1) / 2 ; h$ is a polynomial of degree $m$ in $z$. Thus $z$ belongs to the sub-field $G F^{\prime}\left(2^{m}\right)$ and the order of $z$ is a divisor of $2^{m}-1$. Now $(x+1)^{2}=$ $x^{2}+1=x z$; thus the order of $x^{2}+1$ is a divisor of $p\left(2^{m}-1\right)$ and the order of $x+1$ is the same as that of its square. Again $x(x+1)$ has the same order as $x+1$. Finally $x^{2}+x+1=x(z+1)$ and $z+1$ is also in $G F\left(2^{m}\right)$. Thus the order of all linear and quadratic forms divides $p\left(2^{m}-1\right)<2^{p-1}-1$ for $p>5$.

It is of interest to note that the order of $z$ is precisely $2^{m}-1$ for all suitable primes $p \leqq 139$ except for 37 and 101 for which it is $\frac{1}{3}$ the maximum.

We must, then, seek anong the cubic or higher polynomials for our generators and we turn to a consideration of the computations by which this may be done.

3. The power routine. The primary tool in the investigation is a high-speed routine programmed for SWAC which finds prescribed powers of polynomials $F(x)$ modulis polynomials $G(x)$ and 2 . The routine has two parts and the second part may be used as many times as desired without return to the first. The initial part receives $F^{\prime}(x)$ and $G(x)$ as inputs and computes $n$ successive squares $(F(x))^{2}$, $(F(x))^{4}, \cdots,(F(x))^{2 n}$ where $n$ is the degree of $G(x)$, reducing the results modulis 2 and $G(x)$. The last square is a check; if $G(x)$ is irreducible, $(F(x))^{2 n}=F(x)$. The second receives as input the exponent of the power of $F(x)$ desired, the upper limit being $2^{n}-1$, and computes this power by multiplying together the appropriate stored powers from the first routine. The routines are quadruple precision and, since SIVAC has a 36 bit word, degrees and powers are limited to 143 and $2^{143}-1$ respectively. About $\frac{1}{2}$ second per multiplication is required in either routine. This means a maximum of 75 seconds for completion in either case.

To apply this routine to the problem of determining a primitive generator, $f_{p}(x)$ is used for $G(x)$ and possible generators are used as $F(x)$ in lexicographic succession. The exponents are $\left(2^{p-1}-1\right) / q_{i}$ where $q_{i}$ are the prime divisors of $\left(2^{p-1}-1\right)$. (It is a fortunate circumstance that all necessary factorizations are known [3].) If the power of $F(x)$ is 1 for any of these exponents, $F(x)$ is not a primitive generator, and conversely. As a check the power is also calculated for $q_{i}=1$; here the value must be 1 . Table 1 lists the earliest primitive generator for each prime. 
TABLE 1

\begin{tabular}{c|c} 
Generating polynomials for $G F\left(2^{p-1}\right) ;$ to be used modulis 2 and $\left(x^{2}+1\right) /(x+1)$ \\
\hline 1 & polynomial \\
\hline 3 & $x$ \\
5 & $x+1$ \\
11 & $x^{3}+x+1$ \\
19 & $x^{3}+x+1$ \\
29 & $x^{4}+x+1$ \\
37 & $x^{5}+x+1$ \\
53 & $x^{3}+x^{2}+1$ \\
59 & $x^{4}+x+1$ \\
61 & $x^{3}+x+1$ \\
87 & $x^{3}+x+1$ \\
83 & $x^{5}+x^{2}+1$ \\
101 & $x^{3}+x+1$ \\
131 & $x^{3}+x+1$ \\
139 & $x^{3}+x+1$ \\
& $x^{4}+x^{2}+x+1$ \\
\hline
\end{tabular}

4. Generators of subfields of the cyclotomic fields. There are $\mathbf{1 6}$ primes covered by Table 1 . If subfields are considered, 24 additional fields may be constructed. Specifically, for each polynomial in Table 1 as $F(x)$ and its corresponding $f_{p}(x)$ as $G(x)$ we compute the $\left(2^{p-1}-1\right) /\left(2^{d}-1\right)$-th power of $F(x)$ for each divisor $d$ of $p-1,2<d<p-1$. The results are tabulated in Table 2. Here, in the largest case, the result is a polynomial of degree 138 and we have adopted a condensation of coefficients into octal notation to bring the result within manageable proportions. For example, $x^{7}+x^{5}+x^{4}+x^{2}+x+1$ may be represented first by the ordered octuple of its coefficients: 10110111 , and these may be read in groups of three as octal numbers. Thus the polynomial would appear as 267.

The polynomials of Table 2 are not necessarily the lexicographically earliest generators of their fields. It would be quite impossible to find such generators. However the disadvantages of polynomials of large degree are not as great as might be assumed at first thought. If a number of powers are to be computed, the full size of the registers is needed for the reduction modulo $f_{p}(x)$ and no more additions are needed to compute the irreducible polynomials than if the degrees were smaller. The only unavoidable disadvantage is the danger of an error in transcription. The entries in the table have been preserved against error by a comparison of output decks on successive runs and careful proofreading.

5. Utilization of generators to produce irreducible polynomials. As indicated in section 2, an irreducible polynomial is produced from an element $z$ of $G F\left(2^{n}\right)$ not belonging to a proper subfield by finding the relation of dependence of $z^{0}, z^{1}, z^{2}, \cdots, z^{n}$. In one set of $n+1$ registers are initially stored the powers of $z$. Another companion set is loaded initially with a single 1 in the $i$ th place, $i=0(1) n$. Whenever an operation is performed in one set of registers, the same is done to the companion set. By addition of other elements of the power registers the value 0 is obtained in the $z^{0}$ (or $z^{n}$ ) register. The irreducible polynomial is then read from 
TABLE 2

Generating polynomials for proper sub-fields of $G F\left(2^{-1}\right)$. Fields are $G F\left(2^{d}\right)$ for $d \mid p-1, d \geq 3$. Generators are to be used modulis 2 and $\left(x^{3}+1\right) /(x+1)$. See section 4 for use of octal notation to represent polynomials.

\begin{tabular}{|c|c|c|c|c|c|c|c|c|c|c|c|}
\hline \multirow{2}{*}{$\frac{p}{11}$} & \multirow{2}{*}{$\frac{d}{5}$} & \multicolumn{10}{|c|}{ polynomial } \\
\hline & & & & & & & & & & & 360 \\
\hline 13 & $\begin{array}{l}6 \\
4 \\
3\end{array}$ & & & & & & & & & & $\begin{array}{l}1760 \\
1156 \\
1321\end{array}$ \\
\hline 19 & $\begin{array}{l}9 \\
6 \\
3\end{array}$ & & & & & & & & & $\begin{array}{l}4 \\
1 \\
7\end{array}$ & $\begin{array}{l}77744 \\
26202 \\
63175\end{array}$ \\
\hline 29 & $\begin{array}{r}14 \\
7 \\
4\end{array}$ & & & & & & & & & $\begin{array}{r}10777 \\
6633 \\
17577\end{array}$ & $\begin{array}{l}77705 \\
66330 \\
56617\end{array}$ \\
\hline 37 & $\begin{array}{r}18 \\
12 \\
9 \\
6 \\
4 \\
3\end{array}$ & & & & & & & & $\begin{array}{l}\mathbf{6 4} \\
\mathbf{7 5} \\
\mathbf{3 0} \\
\mathbf{7 1} \\
\mathbf{7 6} \\
\mathbf{7 5}\end{array}$ & $\begin{array}{l}00000 \\
23334 \\
01030 \\
22347 \\
45677 \\
23377\end{array}$ & $\begin{array}{l}00055 \\
20271 \\
20031 \\
11234 \\
73746 \\
31274\end{array}$ \\
\hline 533 & $\begin{array}{r}26 \\
13 \\
1\end{array}$ & & & & & & & $\begin{array}{r}117 \\
117 \\
26\end{array}$ & $\begin{array}{l}77777 \\
42534 \\
50100\end{array}$ & $\begin{array}{l}77777 \\
36165 \\
21003\end{array}$ & $\begin{array}{l}77744 \\
21744 \\
22003\end{array}$ \\
\hline 5!) & 29 & & & & & & & 3777 & 77777 & 77777 & 77760 \\
\hline 61 & $\begin{array}{r}30 \\
20 \\
15 \\
12 \\
10 \\
6 \\
5 \\
4 \\
3\end{array}$ & & & & & & & $\begin{array}{l}17777 \\
17723 \\
17003 \\
34564 \\
55263 \\
14477 \\
76200 \\
36544 \\
10124\end{array}$ & $\begin{array}{l}77777 \\
64723 \\
60103 \\
50135 \\
06377 \\
11710 \\
51450 \\
52035 \\
50147\end{array}$ & $\begin{array}{l}77777 \\
51515 \\
02017 \\
07663 \\
77143 \\
23623 \\
24624 \\
07653 \\
46024\end{array}$ & $\begin{array}{l}77760 \\
17760 \\
00360 \\
71022 \\
15265 \\
74461 \\
01174 \\
31207 \\
52020\end{array}$ \\
\hline 67 & $\begin{array}{r}33 \\
22.2 \\
11 \\
6 \\
3\end{array}$ & & & & & & $\begin{array}{r}64 \\
75 \\
2 \\
25 \\
42\end{array}$ & $\begin{array}{l}00000 \\
24504 \\
13043 \\
46004 \\
11170\end{array}$ & $\begin{array}{l}00000 \\
50000 \\
36044 \\
02150 \\
41201\end{array}$ & $\begin{array}{l}00000 \\
00452 \\
17304 \\
47301 \\
20436\end{array}$ & $\begin{array}{l}00055 \\
35255 \\
32101 \\
41023 \\
22105\end{array}$ \\
\hline N:3 & +1 & & & & & 37 & 77777 & 77777. & 77777 & 77777 & 77760 \\
\hline 101 & $\begin{array}{r}50 \\
25 \\
20 \\
10 \\
5 \\
4\end{array}$ & & & & $\begin{array}{r}1677 \\
1770 \\
1734 \\
620 \\
1000 \\
1667\end{array}$ & $\begin{array}{l}77777 \\
73777 \\
+2260 \\
20714 \\
60310 \\
35170\end{array}$ & $\begin{array}{l}77777 \\
77777 \\
36634 \\
00420 \\
30220 \\
05511\end{array}$ & $\begin{array}{l}77777 \\
71554 \\
35312 \\
64141 \\
00000 \\
25702\end{array}$ & $\begin{array}{l}77777 \\
77777 \\
16067 \\
30210 \\
00220 \\
53322\end{array}$ & $\begin{array}{l}77777 \\
77776 \\
77653 \\
01470 \\
60460 \\
77032\end{array}$ & $\begin{array}{l}77734 \\
70774 \\
36044 \\
20231 \\
30004 \\
10443\end{array}$ \\
\hline 107 & 53 & & & & $37 i \bar{~}$ & 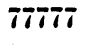 & 7575 & 77770 & 77777 & 77777 & 77760 \\
\hline 131 & $\begin{array}{r}65 \\
26 \\
13 \\
10 \\
5\end{array}$ & & $\begin{array}{r}377 \\
1267 \\
6 \\
1727\end{array}$ & $\begin{array}{r}7775 \\
1634 \\
77723 \\
52020 \\
52023\end{array}$ & $\begin{array}{l}7777 \\
+5062 \\
+5.221 \\
00200 \\
56373\end{array}$ & $\begin{array}{l}77775 \\
04144 \\
15105 \\
22004 \\
62617\end{array}$ & $\begin{array}{l}77777 \\
04004 \\
11634 \\
40100 \\
70360\end{array}$ & $\begin{array}{l}77777 \\
56032 \\
+5045 \\
66020 \\
77+32\end{array}$ & $\begin{array}{l}77777 \\
04432 \\
44225 \\
12043 \\
36763\end{array}$ & $\begin{array}{l}77777 \\
00042 \\
16277 \\
06000 \\
56202\end{array}$ & $\begin{array}{l}77760 \\
36720 \\
77325 \\
00030 \\
57274\end{array}$ \\
\hline 139 & $\begin{array}{r}69 \\
46 \\
23 \\
6 \\
3\end{array}$ & $\begin{array}{l}4 \\
4 \\
4 \\
1 \\
3\end{array}$ & $\begin{array}{l}77777 \\
76637 \\
72055 \\
57550 \\
50006\end{array}$ & $\begin{array}{l}77777 \\
77731 \\
65+24 \\
47372 \\
12434\end{array}$ & $\begin{array}{l}77777 \\
75477 \\
32+52 \\
56617 \\
15601\end{array}$ & $\begin{array}{l}77777 \\
77777 \\
07006 \\
22064 \\
44500\end{array}$ & $\begin{array}{l}77777 \\
77777 \\
07 \pm 14 \\
32472 \\
03000\end{array}$ & $\begin{array}{l}\mathbf{7 7 7 7 7} \\
\mathbf{7 7 7 7 5} \\
\mathbf{0 3 4 1 2} \\
\mathbf{3 6 6 + 1} \\
\mathbf{2} 4144\end{array}$ & $\begin{array}{l}77777 \\
47766 \\
45305 \\
62124 \\
16607\end{array}$ & $\begin{array}{l}77777 \\
37777 \\
06566 \\
04335 \\
05214\end{array}$ & $\begin{array}{l}77744 \\
54744 \\
41345 \\
10117 \\
00270\end{array}$ \\
\hline
\end{tabular}


GalOIS FIELDS OF CHARACTERISTIC TWO AND IRIEDECIBLE POLYNOMIALS 10:3

the companion register. The process can be carried out by systematic diagonalization.

As an example, we construct a primitive irreducible cubic using $z=x^{y}+x^{7}+$ $x^{6}+x^{4}+1(1321)$ for $p=13$. We find $z^{2}=x^{11}+x^{10}+x^{9}+x^{7}+x^{6}+x^{4}+$ $x^{3}+x^{2}$ and $z^{3}=x^{9}+x^{7}+x^{3}+x^{5}$. The calculations can be arranged:

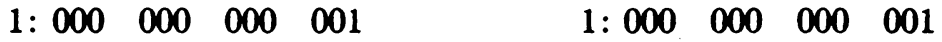

$$
\begin{aligned}
& \begin{array}{lllllllll}
z: & 001 & 011 & 010 & 001 & z^{3}+z: 000 & 000 & 000 & 001
\end{array} \\
& z^{2}: \begin{array}{llll}
111 & 011 & 011 & 100
\end{array} \\
& z^{3}: 001 \quad 011 \quad 010 \quad 000 \\
& z^{3}+z+1: 000 \quad 000 \quad 000 \cdot 000 \text {. }
\end{aligned}
$$

Specifically, $z^{2}$ is used (vacuously) to clear the first column; then discarded. The second column is now empty; $z^{3}$ is used to clear the third column and discarded. All but the last column are now clear and $z^{3}+z$ is used to finish the job; the polynomial is $z^{3}+z+1$. Alternatively we could have started from 1 and the right hand column:

$$
\begin{aligned}
& z+1: 001011010 \quad 000
\end{aligned}
$$

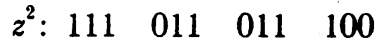

$$
\begin{aligned}
& z^{3}: 001011010 \quad 000, \\
& \begin{array}{cccccccc}
z+1: 001 & 011 & 010 & 000 & z^{3}+z+1: 000 & 000 & 000 & 000
\end{array} \\
& z^{3}: 001 \quad 011 \quad 010 \quad 000
\end{aligned}
$$

The diagonalization process, while requiring large storage, is very rapid. No more than $2 p n^{2}$ additions need be performed.

University of California

Los Angeles, California

1. A. A. Albert, Fundamental Concepls of Higher Algebru, University of Chicago, 1956.

2. L. E. Drcxson, Linear Groups with an Exposition of the Galois Field Theory, Teubner, Leipzig, 1901.

3. I. Kraitchik, Introduction a la theorie des Nombres, Paris, 1952.

4. R. PRICE \& P. E. GREEN, JR., "A communication technique for multipath channels," Proc., IRE, v. 46, 1958 , p. 555 .

5. N. Zierler, "Linear recursive sequences," Soc. Ind. Appl. Math., Jn., v. i, 1959, p. 31. 